\title{
Unitary evolution in Gowdy cosmology
}

\author{
Alejandro Corichi, ${ }^{1,2}$ Jerónimo Cortez, ${ }^{3}$ and Guillermo A. Mena Marugán ${ }^{3}$ \\ ${ }^{1}$ Instituto de Matemáticas, UNAM, Apartado Postal 61-3, Morelia, Michoacan 58090, Mexico \\ ${ }^{2}$ Instituto de Ciencias Nucleares, UNAM, Apartado Postal 70-543, Mexico D.F. 04510, Mexico \\ ${ }^{3}$ Instituto de Estructura de la Materia, CSIC, Serrano 121, 28006 Madrid, Spain
}

(Received 27 October 2005; published 6 February 2006)

\begin{abstract}
Recent results on the nonunitary character of quantum time evolution in the family of Gowdy $T^{3}$ spacetimes bring the question of whether one should abandon the most sacred principle of unitary evolution in cosmology. In this work we show that the answer is in the negative. We put forward a full nonperturbative canonical quantization of the polarized Gowdy $T^{3}$ model that implements the dynamics while preserving unitarity. We discuss possible implications of this result.
\end{abstract}

The issue of unitarity in quantum gravity has been of central importance for the past 30 years, ever since the possibility of black holes evaporating suggested that unitary evolution might be violated. However, most of the attention on this issue has been within the semiclassical and minisuperspace scenarios where the gravitational degrees of freedom are at most finite in number. It is then quite natural to investigate the issue of time evolution within the full theory, or at least for models that still possess an infinite number of degrees of freedom. Of particular relevance are cosmological, spatially closed models where no canonical notion of a (asymptotic) unitary time evolution exists (as is the case for asymptotically flat and anti-de Sitter boundary conditions). It is with this in mind that we consider the simplest of all inhomogeneous closed models, namely, the Gowdy $T^{3}$ cosmology.

Since the mid-1970s, the quantization of the Gowdy $T^{3}$ model [1] has received a great deal of attention $[2,3]$. The first preliminary attempts [2-4] to define a quantum theory and extract physics from the model were followed by more detailed analyses $[5,6]$. Considerable progress has recently been achieved in defining a complete quantization of the (sub-)model with linear polarization [7].

The quantization proposed in [7] is based on the fact that the polarized model can be treated as $2+1$ gravity coupled to an axially symmetric, massless scalar field, defined in a manifold whose topology is $T^{2} \times \mathbb{R}^{+}$. More precisely, once the system is (partially) gauge fixed and a choice of internal time is made, the spacetimes are characterized (modulo a remaining global constraint) by a point particle degree of freedom and a free scalar field $\phi$ propagating in a fictitious two-dimensional expanding torus. Thus, the problem of quantization of the local gravitational degrees of freedom reduces to a quantum theory of the scalar field in the fictitious background. The quantum Gowdy $T^{3}$ model is defined by using a representation for $\phi$ on a fiducial Fock space, where the remaining constraint is imposed to get the physical Hilbert space.

Despite this progress, the quantization put forward in [7] has a serious drawback: the dynamics cannot be imple- mented as a unitary transformation, neither on the kinematical [8] nor in the physical [9] Hilbert space. Even though the dynamics can be approximated as much as desired by means of unitary transformations [10], the model is still lacking a unitary operator that represents the genuine time evolution. The failure of unitarity is, in the best of cases [11], a nontrivial complication that impedes the availability of a Schrödinger picture in which dynamics preserves the conventional notion of probability $[9,12]$. The question then arises of whether one should really abandon the concept of unitary evolution or look instead for a different quantization of the model compatible with unitarity. The relevance of this question surpasses the restricted context of the Gowdy cosmology, which can be viewed as a particular arena in which one is addressing the issue. The aim of this work is to show that, opposite any pessimistic perspective, it is possible to achieve a unitary quantum dynamics in the polarized Gowdy cosmology. At least as far as this system is concerned, there is no intrinsic obstruction to the standard probabilistic interpretation of quantum physics (neither in the Heisenberg nor in the Schrödinger picture) in a cosmological scenario.

Let us briefly recall the model introduced in [7], which was essentially constructed starting with a (partially) gauge-fixed system [10] that, modulo a global constraint, consists of a reduced phase space $\Gamma_{r}=\Gamma_{0} \oplus \tilde{\Gamma}$, where $\Gamma_{0}$ and $\tilde{\Gamma}$ admit as respective coordinates a point particle canonical pair $(Q, P)$ and a "field" canonical pair $\left(\phi(t, \theta), P_{\phi}(t, \theta)\right)$. Here, $\phi$ and $P_{\phi}$ are functions of the (internal) time coordinate $t$ and the spatial coordinate $\theta \in S^{1}$. The corresponding reduced Hamiltonian is

$$
H_{r}=\frac{1}{2} \oint d \theta\left(\frac{P_{\phi}^{2}}{t}+t \phi^{\prime 2}\right)
$$

Thus, the point particle degrees of freedom are constants of motion, whereas a nontrivial evolution takes place only in the field sector $\tilde{\Gamma}$. To be more precise, $\phi$ must satisfy the second-order differential equation 


$$
\ddot{\phi}+\frac{\dot{\phi}}{t}-\phi^{\prime \prime}=0
$$

All smooth solutions to (2), that we will generically denote by $\varphi$, can be written as

$$
\varphi(t, \theta)=\sum_{n \in \mathbb{Z}}\left[A_{n} f_{n}(t, \theta)+A_{n}^{*} f_{n}^{*}(t, \theta)\right],
$$

with

$$
f_{0}(t, \theta)=\frac{1-i \ln t}{\sqrt{4} \pi}, \quad f_{n}(t, \theta)=\frac{H_{0}(|n| t)}{\sqrt{8}} e^{i n \theta} \quad n \neq 0 .
$$

The symbol $*$ denotes complex conjugation, $H_{0}$ is the zeroth-order Hankel function of the second kind [13], and in order to guarantee point-wise convergence, the sequence of constant coefficients $\left\{A_{n}\right\}$ must decrease faster than the inverse of any polynomial in $n$ as $n \rightarrow \pm \infty$.

Equation (2) is the Klein-Gordon equation for a massless, axially symmetric, free scalar field propagating in a fictitious background $\left(\mathcal{M} \simeq T^{2} \times \mathbb{R}^{+}, g_{a b}^{(f)}\right)$, where

$$
g_{a b}^{(f)}=-(d t)_{a}(d t)_{b}+(d \theta)_{a}(d \theta)_{b}+t^{2}(d \sigma)_{a}(d \sigma)_{b},
$$

with $t \in \mathbb{R}^{+}$and $\theta, \sigma \in S^{1}[14]$. Hence, we can identify $\tilde{\Gamma}$ with the canonical phase space of the field in this background, while the space $\tilde{S}$ of smooth solutions can be considered as the covariant phase space of this KleinGordon field. Endowing the space $\tilde{S}$ ( $\tilde{\Omega}$ being its symplectic form) with the "natural" $\tilde{\Omega}$-compatible complex structure $\tilde{J}[7]$,

$$
\tilde{J}\left(\bar{f}_{n}\right)=i \bar{f}_{n}, \quad \tilde{J}\left(\bar{f}_{n}^{*}\right)=-i \bar{f}_{n}^{*},
$$

where $\bar{f}_{n}(t):=f_{n}(t, \theta) \exp [-i n \theta]$, one can construct the "one-particle" Hilbert space $\tilde{\mathcal{H}}$ and, from it, the symmetric Fock space $\mathcal{F}(\tilde{\mathcal{H}})$ on which the formal field operator is written in terms of creation and annihilation operators [corresponding to the positive and negative frequency decomposition defined by the complex structure (4)]. However, the Bogoliubov transformation that implements the dynamics in the quantum theory - by relating at different times either states in $\tilde{\mathcal{H}}$ [8] (Schrödinger picture) or creation and annihilation operators [9] (Heisenberg picture) - turns out not to be square summable in its antilinear part. As a consequence, the evolution dictated by the Hamiltonian (1) fails to be unitarily implementable both at the kinematical level [8] and in the physical Hilbert space [9]. This ends our brief review of the current status of the quantization proposed in [7].

In order to arrive at a unitary theory, we will use the freedom available to redefine the classical phase space through time-dependent canonical transformations. With the resulting set of new canonical variables and its corresponding Hamiltonian, one may then reformulate the quantum Gowdy model. Thus, let us consider the specific canonical transformation [15]:

$$
\begin{gathered}
\bar{Q}:=Q, \quad \bar{P}:=P, \quad \xi:=\sqrt{t} \phi, \\
P_{\xi}:=\frac{P_{\phi}}{\sqrt{t}}+\frac{\phi}{2 \sqrt{t}} .
\end{gathered}
$$

Taking into due account the explicit time dependence of this transformation, the reduced Hamiltonian for the new system of variables becomes

$$
\bar{H}_{r}=\frac{1}{2} \oint d \theta\left(P_{\xi}^{2}+\xi^{\prime 2}+\frac{\xi^{2}}{4 t^{2}}\right) .
$$

Note that this is the Hamiltonian of an axially symmetric, free scalar field with a time-dependent potential that represents an effective mass $1 /(2 t)$, propagating in a fictitious static background $\left(\mathcal{M} \approx T^{2} \times \mathbb{R}^{+}, \bar{g}_{a b}^{(f)}\right)$ with

$$
\bar{g}_{a b}^{(f)}=-(d t)_{a}(d t)_{b}+(d \theta)_{a}(d \theta)_{b}+(d \sigma)_{a}(d \sigma)_{b} .
$$

The Hamiltonian equations derived from (6) lead to

$$
\ddot{\xi}-\xi^{\prime \prime}+\frac{\xi}{4 t^{2}}=0
$$

We will denote by $\zeta$ the smooth solutions to (7), which adopt the generic form

$$
\zeta(t, \theta)=\sum_{n \in \mathbb{Z}}\left[A_{n} g_{n}(t, \theta)+A_{n}^{*} g_{n}^{*}(t, \theta)\right],
$$

where $g_{n}(t, \theta):=\sqrt{t} f_{n}(t, \theta)$, as it is clear from (3) and (5). The complete set of mode solutions $\left\{g_{n}(t, \theta)\right\}$ is "orthonormal" in the product $\left(g_{l}, g_{n}\right)=-i \Omega\left(g_{l}^{*}, g_{n}\right)$ [i.e. $\left.\left(g_{l}, g_{n}^{*}\right)=0,\left(g_{l}, g_{n}\right)=\delta_{l n}=-\left(g_{l}^{*}, g_{n}^{*}\right)\right]$, with [16]

$$
\Omega\left(\zeta_{1}, \zeta_{2}\right)=\oint d \theta\left(\zeta_{2} \partial_{t} \zeta_{1}-\zeta_{1} \partial_{t} \zeta_{2}\right)
$$

Hence, in the field sector, the covariant phase space is the symplectic vector space $S:=(\Omega,\{\zeta\})$, which can equally be coordinatized by the (pairs of complex conjugate) variables $\left\{\left(A_{n}, A_{n}^{*}\right)_{n \in \mathbb{Z}}\right\}$.

Alternatively, we can consider the canonical phase space $\Gamma$, coordinatized by the set of (complex) canonically conjugate pairs $\left\{\left(\xi_{n}, P_{\xi}^{-n}\right)_{n \in \mathbb{Z}}\right\}$, where $\xi_{n}$ and $P_{\xi}^{n}$ are the (implicitly time-dependent) Fourier coefficients of the configuration and momenta of the massive scalar field. Let us now introduce the following transformations for the zero and nonzero modes, respectively:

$$
\begin{array}{cc}
b_{0}=\frac{\xi_{0}+i P_{\xi}^{0}}{\sqrt{2}}, & b_{0}^{*}=\frac{\xi_{0}-i P_{\xi}^{0}}{\sqrt{2}}, \\
b_{n}=\frac{|n| \xi_{n}+i P_{\xi}^{n}}{\sqrt{2|n|}}, & b_{-n}^{*}=\frac{|n| \xi_{n}-i P_{\xi}^{n}}{\sqrt{2|n|}} .
\end{array}
$$

They are canonical, inasmuch as $\left\{b_{n}, i b_{m}^{*}\right\}=\delta_{n m}$. So, one can adopt as coordinates for $\Gamma$ the (complex conjugate) variables $\left\{\left(b_{n}, b_{n}^{*}\right)_{n \in \mathbb{Z}}\right\}$. 
The map from $S$ to $\Gamma$ is given by

$$
\begin{aligned}
& b_{0}(t)=r_{0}(t) A_{0}+s_{0}(t) A_{0}^{*}, \\
& b_{n}(t)=c\left(x_{n}\right) A_{n}+d\left(x_{n}\right) A_{-n}^{*}
\end{aligned}
$$

(for the zero and nonzero modes), where

$$
\begin{aligned}
& s_{0}(t)=\sqrt{\pi} g_{0}^{*}(t, \theta)\left(1+\frac{i}{2 t}\right)-\frac{1}{2 \sqrt{t}}, \\
& r_{0}(t)=2 \sqrt{\pi} g_{0}(t, \theta)-s_{0}^{*}(t),
\end{aligned}
$$

and

$$
\begin{aligned}
& d\left(x_{n}\right)=\sqrt{\frac{\pi x_{n}}{8}}\left[\left(1+\frac{i}{2 x_{n}}\right) H_{0}^{*}\left(x_{n}\right)-i H_{1}^{*}\left(x_{n}\right)\right], \\
& c\left(x_{n}\right)=\sqrt{\frac{\pi x_{n}}{2}} H_{0}\left(x_{n}\right)-d^{*}\left(x_{n}\right) .
\end{aligned}
$$

Here, $x_{n}:=|n| t$ and $H_{1}$ is the first-order Hankel function of the second kind [13]. It is not difficult to see that

$$
\left|r_{0}(t)\right|^{2}-\left|s_{0}(t)\right|^{2}=1, \quad\left|c\left(x_{n}\right)\right|^{2}-\left|d\left(x_{n}\right)\right|^{2}=1,
$$

for all $t>0$. This reflects the fact that (9) is a Bogoliubov transformation between annihilationlike and creationlike variables. Moreover, it can be shown that this timedependent canonical transformation is generated by the Hamiltonian (6). From (9), it follows that in the canonical phase space a state $\left(b_{n}\left(t_{0}\right), b_{n}^{*}\left(t_{0}\right)\right)$ at time $t_{0}$ evolves to the state $\left(b_{n}(t), b_{n}^{*}(t)\right)$ at time $t$ according to

$$
b_{n}(t)=\alpha_{n}\left(t, t_{0}\right) b_{n}\left(t_{0}\right)+\beta_{n}\left(t, t_{0}\right) b_{-n}^{*}\left(t_{0}\right),
$$

where, for the nonzero modes,

$$
\begin{aligned}
& \alpha_{n}\left(t, t_{0}\right)=c\left(x_{n}\right) c^{*}\left(x_{n}^{0}\right)-d\left(x_{n}\right) d^{*}\left(x_{n}^{0}\right), \\
& \beta_{n}\left(t, t_{0}\right)=d\left(x_{n}\right) c\left(x_{n}^{0}\right)-c\left(x_{n}\right) d\left(x_{n}^{0}\right),
\end{aligned}
$$

with $x_{n}^{0}:=|n| t_{0}$. For the zero modes, one gets a similar expression, with the functions $c$ and $d$ substituted by $r_{0}$ and $s_{0}$, and the arguments $x_{n}$ and $x_{n}^{0}$ replaced with $t$ and $t_{0}$. We note that $\left|\alpha_{n}\left(t, t_{0}\right)\right|^{2}-\left|\beta_{n}\left(t, t_{0}\right)\right|^{2}=1$ for all $n$, as it should be because the map (10) on $\Gamma$ is given by the composition of two Bogoliubov transformations and therefore is itself a transformation of this kind.

Taking a fixed time $t_{0}>0$ and considering the inverse of (9), expression (8) can be written in terms of a new "orthonormal" set of solutions $\left\{G_{n}(t, \theta)\right\}$ :

$$
\zeta(t, \theta)=\sum_{n \in \mathbb{Z}}\left[G_{n}(t, \theta) b_{n}\left(t_{0}\right)+G_{n}^{*}(t, \theta) b_{n}^{*}\left(t_{0}\right)\right] .
$$

Explicitly, for the zero and nonzero modes,

$$
\begin{aligned}
G_{0}(t, \theta) & =\sqrt{t}\left[r_{0}^{*}\left(t_{0}\right) f_{0}(t, \theta)-s_{0}^{*}\left(t_{0}\right) f_{0}^{*}(t, \theta)\right], \\
G_{n}(t, \theta) & =\sqrt{\frac{t}{8}}\left[c^{*}\left(x_{n}^{0}\right) H_{0}\left(x_{n}\right)-d^{*}\left(x_{n}^{0}\right) H_{0}^{*}\left(x_{n}\right)\right] e^{i n \theta} .
\end{aligned}
$$

Using the fact that the solutions in (11) are decomposed in complex conjugate pairs, we define a $\Omega$-compatible complex structure $J$ as

$$
J\left(\bar{G}_{n}(t)\right)=i \bar{G}_{n}(t), \quad J\left(\bar{G}_{n}^{*}(t)\right)=-i \bar{G}_{n}^{*}(t),
$$

where $\bar{G}_{n}(t):=G_{n}(t, \theta) \exp [-i n \theta]$. With $(\Omega,\{\zeta\}, J)$ we can construct the "one-particle" Hilbert space $\mathcal{H}$ and the associated symmetric Fock space $\mathcal{F}(\mathcal{H})$, which will be the (kinematical) Hilbert space of the quantum theory. Following this prescription, we can introduce the formal field operator $\hat{\zeta}$ in terms of creation and annihilation operators corresponding to the positive and negative frequency decomposition provided by the complex structure $J$ :

$$
\hat{\zeta}(t ; \theta)=\sum_{n \in \mathbb{Z}}\left[G_{n}(t, \theta) \hat{b}_{n}+G_{n}^{*}(t, \theta) \hat{b}_{n}^{\dagger}\right] .
$$

A comparison with (11) shows that we could have obtained this field operator by naively promoting the constants of motion $\left\{b_{n}\left(t_{0}\right), b_{n}^{*}\left(t_{0}\right)\right\}$ in the solution to annihilation and creation operators $\left\{\hat{b}_{n}\left(t_{0}\right)=\hat{b}_{n}, \hat{b}_{n}^{\dagger}\left(t_{0}\right)=\hat{b}_{n}^{\dagger}\right\}$. This can be understood as the Schrödinger picture.

In the Heisenberg picture, time evolution is provided by the Bogoliubov transformation (10). Namely, by calling $\hat{b}_{n}^{(H)}\left(t_{0}\right):=\hat{b}_{n}$, the relation between the annihilation and creation operators at different times $t_{0}$ and $t$ is

$$
\hat{b}_{n}^{(H)}(t)=\alpha_{n}\left(t, t_{0}\right) \hat{b}_{n}^{(H)}\left(t_{0}\right)+\beta_{n}\left(t, t_{0}\right) \hat{b}_{-n}^{(H) \dagger}\left(t_{0}\right) .
$$

Thus, in this picture we get

$$
\hat{\zeta}(t ; \theta)=\frac{1}{\sqrt{4 \pi}} \sum_{n \in \mathbb{Z}} N_{n}\left[e^{i n \theta} \hat{b}_{n}^{(H)}(t)+e^{-i n \theta} \hat{b}_{n}^{(H) \dagger}(t)\right],
$$

where $N_{n}=1 / \sqrt{|n|}$, except for $N_{0}=1$.

Time evolution is unitarily implementable on the (kinematical) Fock space $\mathcal{F}(\mathcal{H})$ if and only if the sequence $\left\{\beta_{n}\right\}$ is square summable [17]. Since $\beta_{n}=\beta_{-n}$, it suffices to analyze the sequence $\left\{\beta_{k}\right\}$ with $k \in \mathbb{N}$. From the largeargument asymptotic expansions of the Hankel functions [13] one can check that, given any fixed $T>0$, the sequence $\{d(k T)\}$ (with $k \in \mathbb{N}-\{0\}$ ) is square summable. In particular, so are $\{d(k t)\}$ and $\left\{d\left(k t_{0}\right)\right\}$. This implies that there exists an integer $k_{0}$ such that both $|d(k t)|$ and $\left|d\left(k t_{0}\right)\right|$ are smaller than the unity for all $k>k_{0}$. Since $|c|^{2}=1+$ $|d|^{2}$, one also has that $|c(k t)|$ and $\left|c\left(k t_{0}\right)\right|$ are smaller than $\sqrt{2}$ for $k>k_{0}$. In this case,

$$
\begin{aligned}
\left|\beta_{k}\left(t, t_{0}\right)\right|^{2} & \leq 2\left(\left|d\left(x_{k}^{0}\right)\right|+\left|d\left(x_{k}\right)\right|\right)^{2} \\
& \leq 4\left(\left|d\left(x_{k}^{0}\right)\right|^{2}+\left|d\left(x_{k}\right)\right|^{2}\right) .
\end{aligned}
$$

The square summability of the sequence $\left\{\beta_{k}\right\}$ follows then from that of $\{d(k t)\}$ and $\left\{d\left(k t_{0}\right)\right\}$.

Time evolution is hence unitarily implementable on the (kinematical) Fock space $\mathcal{F}(\mathcal{H})$. Moreover, the evolution leaves invariant the constraint

$$
\hat{C}=\sum_{k \in \mathbb{N}} k\left(\hat{b}_{k}^{\dagger} \hat{b}_{k}-\hat{b}_{-k}^{\dagger} \hat{b}_{-k}\right),
$$


which quantum mechanically implements the requirement that the total $(\theta-)$ momentum of the field $\xi$ vanish [10]. As a consequence, the dynamics is unitarily implementable not only on $\mathcal{F}(\mathcal{H})$, but also on the physical Hilbert space $\mathcal{F}_{\text {phys }}(\mathcal{H})$, defined as the kernel of the above constraint. Thus, we have achieved a quantization of the Gowdy $T^{3}$ model where physical states (as well as operators) evolve in a unitary way. This is our main result.

In what follows, we discuss some consequences of this quantization and compare it with previous ones. The first remark is that, whereas $\hat{\xi}\left(t_{0} ; \theta\right)$ evolves unitarily to $\hat{\xi}(t ; \theta)$, the formal operator $\hat{\phi}(t ; \theta):=\hat{\xi}(t ; \theta) / \sqrt{t}$ (considered in [7]) does not. Namely, $\hat{\phi}\left(t_{0} ; \theta\right)$ and $\hat{\phi}(t ; \theta)$ are not unitarily related. Thus, a suitable choice of the fundamental field seems very important for a consistent quantization. In making this choice, we have employed the freedom available to redistribute the time dependence [via the timedependent transformation (5)] in an implicit part, generated by the reduced Hamiltonian of the model, and an explicit part (the factor $1 / \sqrt{t}$ in $\phi$ ), whose time variation does not need to be described by means of a unitary transformation. Note also that it is natural to consider timedependent canonical transformations in the Gowdy model, since the reduced Hamiltonian obtained by gauge fixing is already explicitly time dependent.

Let us now explore the new description proposed here from the viewpoint of the classical structure needed to find the quantum representation. It is known [18] that evolution between two Cauchy surfaces, say $t=t_{1}$ and $t=t_{2}$, can be described on the space of solutions $S$ by a uniparametric family of symplectic maps $\mathcal{T}_{t_{1}, T}$, with $T \in\left[t_{1}, t_{2}\right]$. We can consequently obtain a uniparametric family of induced complex structures $J_{T}=\mathcal{T}_{t_{1}, T} J \mathcal{T}_{t_{1}, T}^{-1}$. In fact, since the dynamics is unitarily implementable, $J_{T}-J$ is HilbertSchmidt (HS) on $\mathcal{H}$ for every element of this family. Similarly, for the description discussed in [7] in terms of the field $\phi$, one gets a family $\tilde{J}_{T}=\tilde{\mathcal{T}}_{t_{1}, T} \tilde{\mathcal{T}} \tilde{\mathcal{T}}_{t_{1}, T}^{-1}$ on $\tilde{S}$. However, $\tilde{J}_{T}-\tilde{J}$ fails to be HS on $\tilde{\mathcal{H}}$ for $T \neq t_{1}$. In addition, recall that the correspondence $\varphi=\zeta / \sqrt{t}$ defines a map $R: S \rightarrow \tilde{S}$ between both spaces of solutions. We can then consider the complex structures $J_{T}^{\prime}=R J_{T} R^{-1}$ induced on $\tilde{S}$ by this map, and ask whether $J_{T}^{\prime}-\tilde{J}_{T}$ is HS on $\tilde{\mathcal{H}}$. It is easy to show that this happens only for $T=t_{1}$ (i.e., for $R J R^{-1}$ and $\tilde{J}$ ). Therefore, even when the complex structures $J$ and $\tilde{J}$ are $(R)$ equivalent, they do not connect unitarily equivalent theories in the explained sense, because the evolution is unitary in one case (in the quantum theory with fundamental field $\xi$ ) but not in the other (in the quantization in which $\phi$ is viewed as the fundamental field). It would also be interesting to explore different quantum representations for each of these choices of fundamental fields and study their equivalence and the unitary implementability of the dynamics [19].

Our results are worthy of some specific comments from the perspective of quantum field theory in curved spacetimes. The Gowdy model was described in [7] in terms of a free massless scalar field $\phi$ on a flat, but time-dependent background. A conventional quantization of this field leads to a nonunitary theory. By a field redefinition, which involves the time parameter, we have mapped the system into a scalar field $\xi$ subject to a time-dependent potential (interpreted as a time-dependent mass), though now the background is flat and time independent (like threedimensional Minkowski spacetime, except for the topology). The natural quantization of this new field that we have put forward provides a theory in which the dynamics is unitary. Besides, one can check that $J_{T}$ approaches, for asymptotically large values of $T$, the (analog of the) Poincaré-invariant complex structure of Minkowski spacetime in the limit in which the system becomes massless. Note that it is only in that limit that the system is invariant under time translations. This result points towards a possible connection between unitary implementability and asymptotic symmetries, but the matter certainly calls for a more thorough investigation.

Finally, let us point out that the vacuum of the quantum theory proposed for $\xi$ is not left invariant by the time evolution, as can be seen either from the presence of the time-dependent potential in the reduced Hamiltonian or from the fact that the induced complex structures $J_{T}$ differ from $J$ except at $T=t_{1}$. The quantum description provides in this way a Hilbert space of physical states $\mathcal{F}_{\text {phys }}(\mathcal{H})$, obtained from a kinematical Fock space $\mathcal{F}(\mathcal{H})$, in which the vacuum evolves approaching the "Poincaré-invariant" vacuum associated with the asymptotic region at large times. This evolution may be interpreted as a production of "particles" by the vacuum $[19,20]$. Nevertheless, no conflict arises for unitarity. In conclusion, we have succeeded in constructing a consistent quantization of the polarized Gowdy model in which, on the one hand, a notion of vacuum that displays a cosmological evolution is available and, on the other hand, dynamics is unitary. This is the first cosmological model with an infinite number of degrees of freedom for which a quantization with these features has been constructed.

This work was supported by the Spanish MEC Project No. FIS2004-01912 and No. HP2003-0140, by CONACyT (México) 40035-F and U47857-F, and by the DGAPAUNAM Grant No. IN108103. J.C. was funded by the Spanish MEC, No./Ref. SB2003-0168. 


\section{UNITARY EVOLUTION IN GOWDY COSMOLOGY}

[1] R. H. Gowdy, Ann. Phys. (N.Y.) 83, 203 (1974).

[2] C. W. Misner, Phys. Rev. D 8, 3271 (1973); B. K. Berger, Phys. Rev. D 11, 2770 (1975).

[3] B. K. Berger, Ann. Phys. (N.Y.) 83, 458 (1974).

[4] B. K. Berger, Ann. Phys. (N.Y.) 156, 155 (1984).

[5] V. Husain and L. Smolin, Nucl. Phys. B327, 205 (1989).

[6] G. A. Mena Marugán, Phys. Rev. D 56, 908 (1997).

[7] M. Pierri, Int. J. Mod. Phys. D 11, 135 (2002).

[8] A. Corichi, J. Cortez, and H. Quevedo, Int. J. Mod. Phys. D 11, 1451 (2002).

[9] C. G. Torre, Phys. Rev. D 66, 084017 (2002).

[10] J. Cortez and G. A. Mena Marugán, Phys. Rev. D 72, 064020 (2005).

[11] For a more pessimistic view of the implications of this problem see e.g. [8].

[12] T. Jacobson, in Conceptual Problems of Quantum Gravity, edited by A. Ashtekar and J. Stachel (Birkhäuser, Boston, 1991).

[13] Handbook of Mathematical Functions, edited by M. Abramowitz and I. A. Stegun (Dover, New York, 1970), 9th ed.
PHYSICAL REVIEW D 73, 041502 (2006)

[14] Note that the field is invariant under a $U(1)$ spatial symmetry, since it depends on the angular coordinate $\theta$.

[15] The change $\xi=\sqrt{t} \phi$ was already considered in [3], but just within the study of the WKB regime.

[16] This symplectic form can be obtained, e.g., from the action functional of the model once the canonical transformation (5) has been performed, and is independent of the constant- $t$ section in which it is evaluated.

[17] D. Shale, Trans. Am. Math. Soc. 103, 149 (1962); R. Honegger and A. Rieckers, J. Math. Phys. (N.Y.) 37, 4292 (1996).

[18] C.G. Torre and M. Varadarajan, Classical Quantum Gravity 16, 2651 (1999).

[19] A. Corichi, J. Cortez, and G. A. Mena Marugán (to be published).

[20] It is worth pointing out that our quantization scheme is radically different from any kind of instantaneous diagonalization. The explicit time dependence of both the canonical transformation (5) and the reduced Hamiltonian (6) has been taken into full account. 